\title{
MSAS Physical Symptom Subscale
}

National Cancer Institute

\section{Source}

National Cancer Institute. MSAS Physical Symptom Subscale. NCI Thesaurus. Code C125363.

A subscale of the Memorial Symptom Assessment Scale that is the average of the frequency, severity, and distress associated with the symptoms of lack of appetite, lack of energy, pain, feeling drowsy, constipation, dry mouth, nausea, vomiting, change in taste, weight loss, feeling bloated, and dizziness. 